ADDENDUM

https://doi.org/10.1038/s41586-018-0722-x

\section{Addendum: The Cancer Cell Line Encyclopedia enables predictive modelling of anticancer drug sensitivity}

Jordi Barretina, Giordano Caponigro, Nicolas Stransky, Kavitha Venkatesan, Adam A. Margolin, Sungjoon Kim, Christopher J. Wilson, Joseph Lehár, Gregory V. Kryukov, Dmitriy Sonkin, Anupama Reddy, Manway Liu, Lauren Murray, Michael F. Berger, John E. Monahan, Paula Morais, Jodi Meltzer, Adam Korejwa, Judit Jané-Valbuena, Felipa A. Mapa, Joseph Thibault, Eva Bric-Furlong, Pichai Raman, Aaron Shipway, Ingo H. Engels, Jill Cheng, Guoying K. Yu, Jianjun Yu, Peter Aspesi Jr, Melanie de Silva, Kalpana Jagtap, Michael D. Jones, Li Wang, Charles Hatton, Emanuele Palescandolo, Supriya Gupta, Scott Mahan, Carrie Sougnez, Robert C. Onofrio, Ted Liefeld, Laura MacConaill, Wendy Winckler, Michael Reich, Nanxin Li, Jill P. Mesirov, Stacey B. Gabriel, Gad Getz, Kristin Ardlie, Vivien Chan, Vic E. Myer, Barbara L. Weber, Jeff Porter, Markus Warmuth, Peter Finan, Jennifer L. Harris, Matthew Meyerson, Todd R. Golub, Michael P. Morrissey, William R. Sellers, Robert Schlegel \& Levi A. Garraway

Addendum to: Nature https://doi.org/10.1038/nature11003, published online 28 March 2012; addendum 28 November 2012.

The Supplementary Information of our original Letter (https:// doi.org/10.1038/nature11003) and the Addendum published on 28 November 2012 (https://doi.org/10.1038/nature11735; hereafter referred to as 'first Addendum') contain all of the data used for our analyses. These data include normalized activity (growth inhibition) responses for every cell line at each dose for all 24 of the compounds and drugs tested. These activity values were calculated from replicated data points extracted from one or more experimental assay plates, and normalized using treated and untreated controls from the same plates; as detailed in the original Letter (see the original Supplementary Information 2, page 10), and in the first Addendum. In subsequent correspondence, readers expressed an interest in reproducing our experimental pre-processing, so we shared with them the raw Cell Titer Glo (CTG) data values from each experimental well. These data are now provided as a reference for future readers.
Supplementary Data 1 to this Addendum contains raw values from wells with cell lines growing under control (AC, positive active; NC, untreated negative) or drug-treated (SA, single-agent) conditions. Supplementary Data 1 contains two row entries per well (one with raw CTG readouts, and the other with calculated activity values), and each column contains descriptions or response values, as detailed in Table 1 of this Addendum. Only valid wells are included, so there may be missing concentrations from curves containing data that were flagged as 'invalid'. Compound and cell line names in Supplementary Data 1 are as they were originally captured, which in some cases may differ from names used in the Supplementary Tables of the original Letter and first Addendum. Calculated activity values may also differ in some cases from those in the original Letter, owing to subsequent quality-control call changes in our database since 2012. Chemical names can be reconciled using Table 2 of this Addendum, noting that 'sample-ID' denotes the 'ID-concept' of Table 2 with salt form and batch suffixes appended. Discrepant cell line names may be understood with reference to columns A, C and D of the 'All_Drug_response_data' sheet in supplementary data 1 from the 2015 CCLE and GDSC consortium paper ${ }^{1}$.

We thank Jessie Ambrose and Vladimir Baranov for extracting and quality-checking the raw chemical response data in this Addendum. We also thank the reader who requested the raw data and assisted in quality-checking these results.

1. The Cancer Cell Line Encyclopedia Consortium \& The Genomics of Drug Sensitivity in Cancer Consortium. Pharmacogenomic agreement between two cancer cell line data sets. Nature 528, 84-87 (2015).

Table 1 | Descriptions for key columns in Supplementary Data 1

\begin{tabular}{ll}
\hline Header & Description \\
\hline CELL_LINE_NAME & Cell line name \\
MASTER_PLATE_NAME & Name of master plate for compound dispensing \\
PLATE_GROUP & Experimental batch identifier \\
ASSAY_PLATE_NAME & Barcode identifier for this experimental plate \\
DATE_CREATED & Date and time of experimental plate readout \\
LAYER_TYPE & CTG readout (RAW) or calculated activity (OUTPUT) \\
WELL_TYPE & Untreated (NC), positive (AC), or drug treated (SA) \\
COLUMN_ID & Column identifier for this experimental well \\
ROW_ID & Row identifier for this experimental well \\
COMPOUND & Sample-ID for the chemical used \\
CONCENTRATION & Compound concentration in micromoles \\
VALUE & Raw CTG readout value \\
\hline
\end{tabular}


Table 2 | Chemical name mappings

\begin{tabular}{|c|c|c|c|c|}
\hline Name & ID-concept & Name-probe & Name-generic & Name-brand \\
\hline paclitaxel & $\begin{array}{l}\text { NVP-LEE850; NVP-BGT136; NVP- } \\
\text { LLP829; CHIR167540 }\end{array}$ & TL-139; BMS-181339; LSM-1102; MGI-GP; SW198621 & paclitaxel & $\begin{array}{l}\text { Taxol; Genexol; } \\
\text { Abraxane }\end{array}$ \\
\hline irinotecan & NVP-LBN777; CHIR169217 & $\begin{array}{l}\text { CPT-11; U-101440E; CPT11; LSM-2167; DQ-2805; } \\
\text { NSC-616348; SW197790; SW199156 }\end{array}$ & irinotecan & $\begin{array}{l}\text { Camptosar; Campto; } \\
\text { Topotecin }\end{array}$ \\
\hline topotecan & $\begin{array}{l}\text { NVP-LBN564; NVP-LBN816; } \\
\text { CHIR158120; SN-38 }\end{array}$ & $\begin{array}{l}\text { SKF-104864; INOC-010; INX-0076; BRD-A36630025; } \\
\text { 7-ethyl-10- hydroxycamptothecin; LSM-5662; } \\
\text { E-89-001; NK-211; NSC-609699; SKF-S-104864-A; } \\
\text { SW197557; SW199637 }\end{array}$ & topotecan & $\begin{array}{l}\text { Hycamtin; Evotropin; } \\
\text { Topotecan; Hycamptin }\end{array}$ \\
\hline panobinostat & NVP-LBH589 & NVP-LBH589; LSM-4284; LBH-589 & panobinostat & Faridak \\
\hline$L-685458$ & $\begin{array}{l}\text { NVP-LLP590; NVP-AHK537; } \\
\text { CGP055750 }\end{array}$ & L-685458; L-682679; BRD-K87317732 & & \\
\hline tanespimycin & $\begin{array}{l}\text { NVP-LJM835; NVP-BAG500; NVP- } \\
\text { LLP773; CHIR371658 }\end{array}$ & $\begin{array}{l}\text { 17-AAG; CNF-1010; IPI-493; IPI-504; KOS-953; LSM- } \\
\text { 5870; BRD-K81473043; CP-127374; NSC-330507; } \\
\text { NSC-704057 }\end{array}$ & $\begin{array}{l}\text { tanespimycin; } \\
\text { retaspimycin; } \\
\text { 17-allylaminogel- } \\
\text { danamycin }\end{array}$ & \\
\hline NVP-LBW242 & NVP-LBW242; NVP-LDK934 & $\begin{array}{l}\text { CHEMBL1950720; SureCN2731929;NVP-LBW242; } \\
\text { LBW242 }\end{array}$ & & \\
\hline nutlin-3A & NVP-BEP453; & LSM-6351 & nutlin-3A & \\
\hline palbociclib & NVP-LCY393 & PD-0332991; LSM-1071 & palbociclib & \\
\hline saracatinib & NVP-BHT496 & AZD-0530; NSC-735464; KIN001-045; LSM-1032 & saracatinib & \\
\hline PLX-4720 & NVP-LFL230; CHIR779209 & PLX-4720; LSM-1049 & & \\
\hline PD-0325901 & NVP-LBW624; CHIR371086 & $\begin{array}{l}\text { PD-0325901; DB07046; CHEMBL447345; CHEM- } \\
\text { BL573579; CHEMBL573819; LSM-1101 }\end{array}$ & & \\
\hline selumetinib & NVP-LFE158 & $\begin{array}{l}\text { AZD-6244; Arry-142886; LSM-1056; BRD-K57080016; } \\
\text { ARRY-886 }\end{array}$ & selumetinib & \\
\hline NVP-RAF265 & $\begin{array}{l}\text { NVP-RAF265; HIR371265; } \\
\text { HIR565852 }\end{array}$ & CHIR-265; NVP-RAF265; LSM-1207 & & \\
\hline nilotinib & NVP-AMN107; NVP-LOY196 & NVP-AMN107; LSM-1099;AMN-107 & nilotinib & Tasigna \\
\hline NVP-TAE684 & NVP-TAE684 & NVP-TAE684; TAE-684; KIN001-017; LSM-1024 & & \\
\hline crizotinib & NVP-LDQ718; NVP-BQK827 & PF-2341066; KIN001-023; LSM-1027 & crizotinib & Xalkori \\
\hline erlotinib & $\begin{array}{l}\text { NVP-XBX005; CHIR381147; } \\
\text { CGP084057; CGP084057A }\end{array}$ & $\begin{array}{l}\text { CP-258; OSI-774; RG-1415; Ro-50-8231; LSM-1097; } \\
\text { CP-358774; NSC-718781; R-1415; SW198886 }\end{array}$ & erlotinib & Tarceva \\
\hline lapatinib & NVP-BCZ548 & $\begin{array}{l}\text { GW-2016; GW-572016F; GSK-572016; LSM-1051; } \\
\text { W-572016; SW199101 }\end{array}$ & lapatinib & Tykerb; Tyverb \\
\hline dovitinib & $\begin{array}{l}\text { NVP-TKI258; NVP-BAV666; } \\
\text { CHIR154258 }\end{array}$ & CHIR-258; TKI-258; NVP-TKI258; LSM-1127 & dovitinib & \\
\hline sandostatin & NVP-AES222; NVP-AEW541 & NVP-AEW541; CC-535; LSM-1122; AEW541 & sandostatin & \\
\hline PHA-665752 & NVP-BBD023 & $\begin{array}{l}\text { PHA-665752; SureCN140412; TCMDC-125885; LSM- } \\
1125\end{array}$ & & \\
\hline vandetanib & NVP-AFD094; CHIR160840 & ZD-6474; AZD-6474; LSM-1199 & vandetanib & Caprelsa; Zactima \\
\hline sorafenib & NVP-LBK294; CHIR156503 & Bay-43-9006; NSC-724772; LSM-1008 & sorafenib & Nexavar \\
\hline
\end{tabular}

